\title{
VARIABLE RADIO SOURCES: \\ COMPARISON OF OBSERVATIONS WITH \\ THE ADIABATIC SPHERICAL EXPANSION SOURCE MODEL
}

\author{
EUGENE E. EPSTEIN \\ Aerospace Corporation, Los Angeles, Calif., U.S.A.
}

\begin{abstract}
The adiabatic spherical expansion model of radio source outbursts has been applied to several outbursts in the quasi-stellar sources 3C 273 and $3 C 454.3$ and the Seyfert galaxies $3 \mathrm{C} 84$ and $3 \mathrm{C} 120$. Considering the simplicity of the model, it represents the data exceedingly well in a number of outbursts. The times of origin of the outbursts, the electron energy distribution indices, and the acceleration of the expansion parameters have been derived. Estimates of the sizes and magnetic fields of the outbursts have been made.

These results are part of a paper to be submitted to the Astrophys. $J$.
\end{abstract}

\section{Discussion}

van der Laan: The expressions which Dr Epstein used to compare data and theory are from the model which is instantaneous, homogeneous, spherically symmetric and non-relativistic. We never expected this model to be conformed to at wavelengths shorter than a few $\mathrm{mm}$. When therefore the $3 \mathrm{~mm}$ flux is below the value deduced from longer wavelength data one need not look for a way of quenching the electrons radiating at that wavelength, because it is likely that there the instantaneous approximation is invalid; the generation duration overlaps the decay period computed at the instant of observation. The so-called poor fit at $3 \mathrm{~mm}$ is therefore more informative than the good fits mentioned.

As for developing more sophisticated models, there is a lack of input for this work, since the variables conform to the simple model discouragingly well. It is important therefore to continue monitoring sources which do not fit the model, in both intensity and polarization. 